\title{
Research on Extraction and Properties of Antler Plate Collagen Protein \\ Yao $\mathrm{Na}$ \\ College of Life Sciences, Engineering Research Center of the Chinese Ministry of Educationfor \\ Bioreactor and Pharmaceutical Development, Jilin Agricultural University,130118
}

Key Words: antler plate, collagen protein, protease

\begin{abstract}
As far as antler plate is concerned, we adopt pepsin extraction method to extract the collagen protein in antler plate and use the vacuum freeze to dry into antler plate collagen protein powder in the paper. In addition, we adopt the biological enzymolysis technology to make antler plate collagen protein powder be enzyme digested into peptide. From the perspective of improving the utilization ratio of antler plate collagen protein we start to reduce the costs and improve the competitiveness of the enterprises. The collagen protein is the main component of vertebrates protein. We mainly investigate the influence of different concentrations (1\%, $2 \%, 3 \%, 4 \%, 5 \%)$ of pepsin on the collagen protein extraction, and we examine the in-vitro fiber capacity of collagen protein and denatured temperature of collagen protein.
\end{abstract}

\section{Experimental Materials}

\subsection{Materials and Reagents}

Experimental materials: The antler plate is provided by Jilin Luye Co., $\quad$ Ltd. First we use the meat and bone grinder to shatter the antler plate. Then the antler plate is shattered into 160 mesh with the medicinal herb grinder and it is kept in the refrigerator. The reagents used in the experiment can be shown in Table 1.

Table 1

experimental

reagents

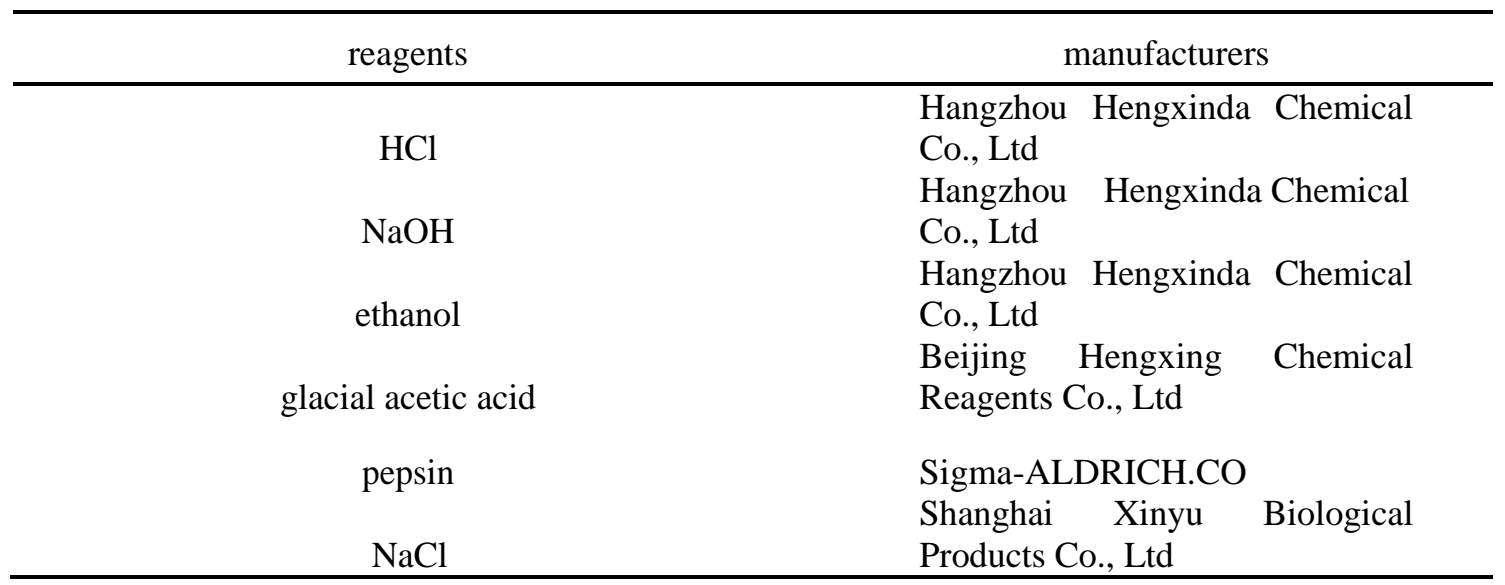

\subsection{Experimental Instruments}

Main experimental instruments can be shown in Table 2. 
Table 2

experimental

instruments

\begin{tabular}{ccc}
\hline instruments names & instruments types & manufacturers \\
\hline electronic balance & AL204 & $\begin{array}{c}\text { Yuanhuaxing Technology Co., Ltd } \\
\text { Beijing Huaxing Technology } \\
\text { Development Co., Ltd. }\end{array}$ \\
freeze drier & LGJ-30 & Shanghai Yiheng Instruments Co., Ltd \\
drying oven & PH-050A & Yuanhuaxing Technology Co., Ltd \\
thermostat water bath & DZKW &
\end{tabular}

\section{Experimental Methods}

\subsection{Antler Plate Degreasing}

We add the scattered antler plate protein into the $10 \%$ acetone with 20 times volume and put it under the magnetic stirrer to stir for 2 days(2d) so as to remove the fat and pigment. We replace the degreasing agent every 12 hours( $12 \mathrm{~h}$ ) and put it into the centrifuge (2000 r/min, $20 \mathrm{~min}$ ) so as to take the precipitation as the standby.

\subsection{Antler Plate Decalcification}

We add the $0.5 \mathrm{mom} /$ acetic acid with 10 times volume into the sediment after the grease removal, and mix and wash for 2 days(2d) over and over again. We remove the supernatant with centrifugation and wash the residue with distilled water and adjust $\mathrm{PH}$ to be neutral.

\subsection{Protein Elimination}

We add $0.1 \mathrm{~mol} / \mathrm{l} \mathrm{NaOH}$ with the volume ratio of $1: 10$ into the sediment after calcium removal and mix and wash for 2 days(2d) over and over again. We remove the supernatant with centrifugation and wash the residue with distilled water and adjust $\mathrm{PH}$ to be neutral.

\subsection{Collagen Protein Extraction}

We extract the collagen protein from the antler plate pretreated well by adopting the pepsin with different concentrations and $0.5 \mathrm{~mol} / \mathrm{l}$ acetate buffer with ten times volume in this experiment. We make the extracting solution be centrifuged with $6000 \mathrm{r} / \mathrm{min}$ for $40 \mathrm{~min}$. Then we collect the supernatant and slowly add $1 \mathrm{~mol} / \mathrm{l} \mathrm{NaOH}$ till $\mathrm{pH}=7.0$, and put it for $24 \mathrm{~h}$, and put it into the centrifugal machine. Then we use $0.5 \mathrm{~mol} / \mathrm{l}$ acetate buffer to adjust $\mathrm{PH}$ to 3.5 , then we add the sodium chloride solid till the final concentration becomes $0.9 \mathrm{~mol} / \mathrm{l}$. We put it in the temperature of $4{ }^{\circ} \mathrm{C}$ for 24 hours(24h), and make it be centrifuged with $6000 \mathrm{r} / \mathrm{min}$ for $40 \mathrm{~min}$. We collect the sediments to freeze and dry so as to obtain antler plate collagen protein powder and keep it in the refrigerator.

\subsection{Calculation of Collagen Protein Concentration}

Hydroxyproline is the characteristic amino acid of collagen protein. Measurement of the content of hydroxyproline after collagen hydrolysis is the most accurate method to do the quantitative analysis of collagen protein. In the $550 \mathrm{~nm}$ we measure the light absorption value.

\subsection{SDS-PAGE Electrophoresis}

We adopt SDS-PAGE electrophoresis to measure antler plate collagen hydrolysate molecular weight. The specific operation steps can be shown as follows. We need to prepare the reagents needed by us well in advance for standby. We install the electrophoresis tank with notch outward. We prepare separation gel to add acrylamides into small beaker with $10 \mathrm{ml}$ one by one, and we usually use the formulation of $10 \mathrm{ml}$. Note: acrylamides are toxic. It is necessary to wear gloves to operate it. $10 \%$ SDS will have the sediments, and we heat them until dissolved. We take TEMED out of the refrigerator, add it into $10 \%$ SDS. After we finish adding it, we use the syringe whose needle has been removed to suck and penetrate once slowly(mixing). Then we use the syringe to suck slowly, and use the syringe needle 
to penetrate into the the slot along the slot wall slowly with the amount of about 7-8ml(slowly operate and prevent the bubbles entering). We add a little distilled water to cover the glue very slowly and wait for gelatinization(Water can prevent the glue shrinking). After the gelatinization, we pour the water away and use the absorbent paper to suck it dry.

$6.5 \%$ concentration glue: We usually prepare it with $5 \mathrm{ml}$ dose proportion. We add ammonium persulfate, insert the comb, and add TEMED again. And we suck and penetrate it with homogeneous mixing according to the above method, and we pour the glues.

Samples treatment:We treat the samples when they are gelatinized. We collect a few samples and add SDS (do not add DTT), and add sulphur-based ethanol with 15-20ul, and heat and boil for 3-5min. Sulphur-based ethanol used in the experiment is undiluted.

Sample adding: After the gelatinization, we take the adhesive tape away, and turn the glues over with the notch inward to put it in the electrophoresis tank, and add some buffer solutions into the glue empty area to get rid of air bubbles with $15 \mu \mathrm{l}$ sample and marker10 $\mu 1$ in each empty area. After we finish adding the samples, we add some buffer solutions slowly. First we add a part of buffer solutions into the tank inside, and we add the remaining buffer solutions into the the tank outside.

Electrophoresis apparatus: We connect the positive and negative wire, and then open the electrophoresis apparatus. The general setting-SN=2( interface) $\mathrm{S}=1 \mathrm{U}=70 \mathrm{~T}=00: 25$ ( spacer gel condition) $\mathrm{S}=2 \mathrm{U}=120 \mathrm{~V} \mathrm{~T}=01: 30$ (separation gel condition, the irregular stripes can reduce $\mathrm{U}$ number) runs to the place with the distance of $0.5 \mathrm{~cm}$, and immediately stops electrophoresis and takes the film out to dye and decolor.

\subsection{In-vitro Original Fiber Production Experiment}

Under the simulated physiological condition of antler plate collagen protein $\left(37^{\circ} \mathrm{C}\right.$, neutral salt solution), we put antler plate collagen protein for two hours to record the change of absorbance in different time intervals( $\lambda=313 \mathrm{~nm})$. The fiber capacity has the positive correlation with its absorbance.

\subsection{UV-VIS Scanning Spectra}

We make the antler plate collagen protein power after lyophilization be dissolved in the distilled water and deployed into $2 \mathrm{mg} / \mathrm{mL}$ solution. We set the control group as the buffer solution, and make the antler plate collagen protein solution deployed well be dissolved in 0.5 insodium acetate, and use UV-VIS to scan in wavelength range from $190 \mathrm{~nm}$ to $400 \mathrm{~nm}$.

\section{Result and Analysis}

\subsection{Effects of Different Enzyme Concentrations on the Extraction Ratio of Collagen Protein}

At room temperature, we add $0.5 \mathrm{M}$ acetate buffer with ten times volume, and conduct the enzymolysis for 12 hours(12h) to investigate respectively the effects of different enzyme concentrations( ( $1 \%, 2 \%, 3 \%, 4 \%$ and $5 \%)$ on the extraction ratio of collagen protein. 


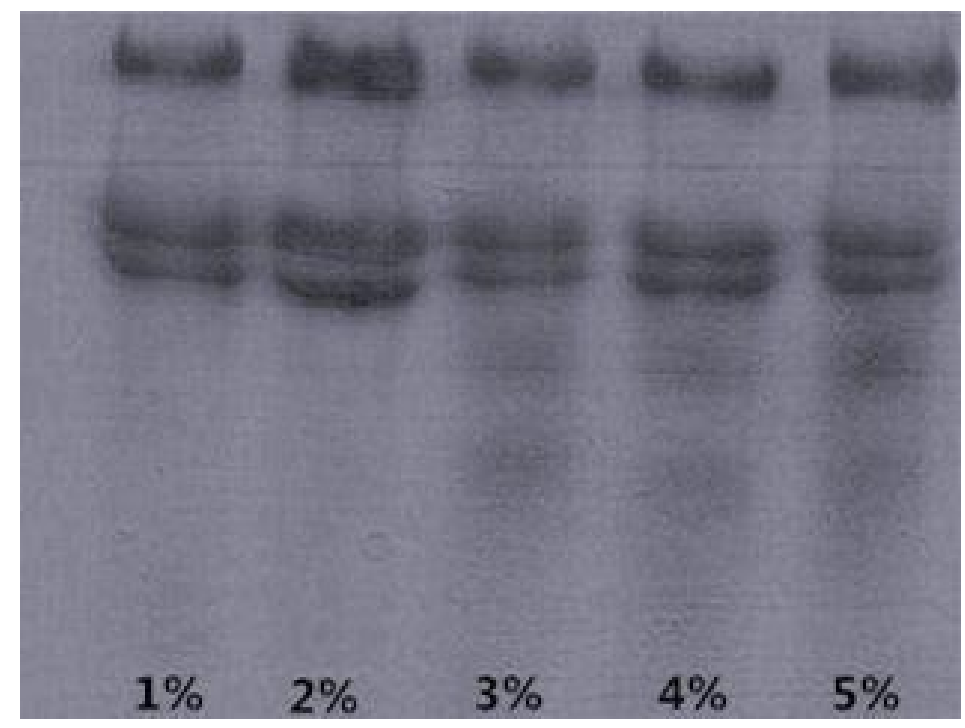

Figure 1 Effects of different pepsin concentrations on stripes of antler plate collagen protein

With the increase of pepsin concentration, the extraction ratio of antler plate collagen protein and pepsin concentration are positively correlated. After pepsin content increases to $2 \%$, the extraction ratio of antler plate collagen protein doesn't change obviously. It shows in Figure 1 that when the volume of enzyme is more than $3 \%$, pepsin has a certain chance to make the collagen be hydrolyzed into small molecular polypeptides. Under the comprehensive consideration, the amount of pepsin selected in the experiment is $2 \%$.

\subsection{Measurement of In-vitro Fiber}

The soluble collagen molecules prepared from animal tissues can form fibrous supramolecular aggregates under in-vitro certain conditions to simulate the appropriate physical conditions, and collagen terminal peptide can be detected in the place of optical density of $313 \mathrm{~nm}$. The absorbance of collage increases quickly in a short period of time. It shows that fiber generation is very quickly. However, with the constant generation of collagenous fiber, absorbance gradually trends to be stable.

\subsection{Ultraviolet Spectrum Scan of Collagen Protein}

Collagen protein contains $\mathrm{COOH}, \mathrm{C}=\mathrm{O}, \mathrm{CONH} 2$ and other chromophoric groups and they can produce the absorption peak. Antler plate collagen protein solution extracted by pepsin has weak absorption peak in the place of $280 \mathrm{~nm}$, and it has the maximal absorption peak in the place of $226 \mathrm{~nm}$. It shows that antler plate collagen protein solution extracted by pepsin contains few amino acids (tryptophan, tyrosine and phenylalanine) contents with benzene ring conjugated double bond in extracted antler plate collagen protein solution. This conforms to characteristics of type I collagen protein in related materials.

\section{Conclusion}

By studying the effects of different enzyme amounts on the extraction rate of antler plate collagen protein we determine the best concentration of antler plate collagen protein solution extracted by pepsin is $2 \%$. Electrophoresis stripes are clearer, there are no small molecule polypeptide stripes.

We conduct the in-vitro fiber and denaturation temperature detection on extracted collagen protein. Thus, it is concluded that the extracted collagen protein has good in-vitro fiber ability. By analyzing UV-VIS spectra map, we can see that antler plate collagen protein solution extracted by 
pepsin has weak absorption peak in the place of $280 \mathrm{~nm}$, and it has the maximal absorption peak in the place of $226 \mathrm{~nm}$. It shows that antler plate collagen protein solution extracted by pepsin contains few amino acids (tryptophan, tyrosine and phenylalanine) contents with benzene ring conjugated double bond in extracted antler plate collagen protein solution. This conforms to characteristics of type I collagen protein in related materials.

\section{References}

[1]Liu Shaohua. Research on the Extraction of Antler Plate Collagen Protein and Angiotensin Converting Enzyme (ACE) Inhibitory Peptide Activity[D]. Jilin Agricultural University, 2015.

[2]Wei Gongqiang, Liu Shaohua, Fanning, Li Liang, Li Huiping, and Zhao Yan. With Response Surface Methodology the Optimization of Sika Deer Antler Plate Collagen Protein Extraction Process[J]. Journal of Economic Animal, 2014, 04:198-205.

[3]Wei Gongqiang, Liu Shaohua, Fanning, Li Liang, Li Huiping, Zhao Yan and Zhang Lianxue. Research on the Optimization of Antler Plate Collagen Protein Enzymolysis Extraction Process with Response Surface Methodology[J]. Journal of Northwest Sci-Tech University of Agriculture and Forestry(Natural Science Edition), 2015, 06:205-214.

[4] Liu Shaohua, Fanning, Li Liang, Li Huiping, Zhao Yan and Wei Gongqiang. With Response Surface Methodology the Optimization of Sika Deer Antler Plate Collagen Protein Extraction Process[J]. Jilin Traditional Chinese Medicine, 2014, 10:1027-1030.

[5]Wei Gongqiang, Liu Shaohua, Fanning, Li Liang, Zhao Yan and Zhang Lianxue. Research on Characteristics of Red Deer Antler Plate Collagen Protein[J]. Shanghai Journal of Traditional Chinese Medicine, 2014, 09:88-92. 